7th International Workshop on Astronomy and

Relativistic Astrophysics (IWARA 2016)

International Journal of Modern Physics: Conference Series

Vol. 45 (2017) 1760031 (8 pages)

(C) The Author(s)

DOI: $10.1142 / \mathrm{S} 201019451760031 \mathrm{X}$

\title{
Maxwell Equation Violation by Density Dependent Magnetic Fields in Neutron Stars
}

\author{
Marcelo D. Alloy \\ Departamento de Ciências Exatas e Educação \\ Universidade Federal de Santa Catarina (UFSC) \\ 89.065-300 Blumenau, SC, Brazil \\ marcelo.alloy@ufsc.br \\ Débora P. Menezes \\ Departamento de Física \\ Universidade Federal de Santa Catarina (UFSC) \\ 88.040-900 Florianópolis, SC, CP 476, Brazil, \\ debora.p.m@ufsc.br
}

Published 15 August 2017

\begin{abstract}
We show that the widely used density dependent magnetic field prescriptions, necessary to account for the variation of the field intensity from the crust to the core of neutron stars violate one of the Maxwell equations. We estimate how strong the violation is when different equations of state are used and check for which cases the pathological problem can be cured. We then propose a simple solution that allows for the usual prescriptions to be used without violating a fundamental equation of physics.
\end{abstract}

Keywords: Equation of State, Neutron Stars, Compact Stars.

PACS numbers: 26.60.+c; 21.65.+f; 97.60.Jd

\section{Introduction and Formalism}

The physics underlying the quantum chromodynamics (QCD) phase diagram has still not been probed at all temperatures and densities. While some aspects can be confirmed either by lattice QCD or experimentally in heavy ion collisions for instance, other aspects depend on extraterrestrial information. One of them is the possible constitution and nature of neutron stars (NS), which are compact objects related to the low temperature and very high density portion of the QCD phase diagram. Astronomers and astrophysicists can provide a handful of information obtained from observations and infer some macroscopic properties, namely NS

This is an Open Access article published by World Scientific Publishing Company. It is distributed under the terms of the Creative Commons Attribution 4.0 (CC-BY) License. Further distribution of this work is permitted, provided the original work is properly cited. 
masses, radii, rotation period and external magnetic fields, which have been guiding the theory involving microscopic equations of state (EOS) aimed to describe this specific region of the QCD phase diagram.

There are different classes of NS and three of them have shown to be compatible with highly magnetised compact objects, known as magnetars, ${ }^{1,2}$ namely, the soft gamma-ray repeaters, the anomalous X-ray pulsars and more recently, the repeating fast radio burst. ${ }^{3}$ The quest towards explaining these NS with strong surface magnetic fields, has led to a prescription that certainly violates Maxwell equations. ${ }^{4}$ The aim of this letter is to show how strong this violation is, to check whether the density dependent magnetic field prescriptions can or cannot be justified and finally to identify in which cases, the density dependent prescriptions can still be used.

Magnetars are likely to bear magnetic fields of the order of $10^{15} \mathrm{G}$ on their surfaces, which are three orders of magnitude larger than magnetic fields in standard NS. In the last years, many papers dedicated to the study of these objects have shown that the EOS are only sensitive to magnetic fields as large as $10^{18} \mathrm{G}$ or stronger. $^{5,6}$ The Virial theorem and the fact that some NS can be quark (also known as strange) stars allow these objects to support central magnetic fields as high as $3 \times 10^{18} \mathrm{G}$ if they contain hadronic constituents and up to $10^{19} \mathrm{G}$ if they are quark stars. To take into account these possibly varying magnetic field strength that increases towards the centre of the stars, the following proposition was made: ${ }^{4}$

$$
\mathbf{B}=B_{z}(n) \mathbf{k}=\left[B^{\text {surf }}+B_{0}\left\{1-\exp \left\{-\beta\left(\frac{n}{n_{0}}\right)^{\gamma}\right\}\right\} \mathbf{k}\right.
$$

where $B^{\text {surf }}$ is the magnetic field on the surface of the neutron stars taken as $10^{14} G$ in the original paper, $n$ is the total number density, and $n_{0}$ is the nuclear saturation density In subsequent papers ${ }^{7-},{ }^{19}$ the above prescription was extensively used, with many variations in the values of $B^{\text {surf }}$, generally taken a $10^{15} \mathrm{G}$ on the surface, $\beta$ and $\gamma$, arbitrary parameters that cannot be tested by astronomical observations. The high degree of arbitrariness was checked already in ${ }^{4}$ and later in ${ }^{20}$ and more than $50 \%$ variation in the maximum stellar mass and $25 \%$ variation in the corresponding radius was found.

With the purpose of reducing the number of free parameters from two to one and consequently the arbitrariness in the results, another prescription was then proposed in: ${ }^{20}$

$$
\mathbf{B}=B_{z}(\epsilon) \mathbf{k}=\left[B^{\text {surf }}+B_{0}\left(\frac{\epsilon}{\epsilon_{c}}\right)^{\alpha}\right] \mathbf{k},
$$

where $\epsilon_{c}$ is the energy density at the center of the maximum mass neutron star with zero magnetic field, $\alpha$ is any positive number and $B_{0}$ is the fixed value of the magnetic field. With this recipe, all magnetic fields converge to a certain value at some large energy density, despite the $\alpha$ value used.

It is important to stress that in most papers, the density dependent expressions are written in such a way that it may lead to the misinterpretation that the functions 
give just the magnitude of the magnetic field and that, in spherical symmetry, the field would depend only on the radial coordinate. However, this interpretation is not consistent with the fact that the very same magnetic field is always assumed to exist only in the $z$ direction. One of the Maxwell equations tells us that $\nabla \cdot \mathbf{B}=0$. In most neutron star calculations, the magnetic field is chosen as static and constant in the $z$ direction, as proposed in the first application to quark matter. ${ }^{5}$ In this case, the energy associated with the circular motion in the $x-y$ plane is quantised (in units of $2 q B, q$ being the electric charge) and the energy along $z$ is continuous. The desired EOS is then obtained and the energy density, pressure and number density depend on the filling of the Landau levels. Apart from the original works, ${ }^{5,6}$ detailed calculations for different models can also be found, for instance, in ${ }^{9,15}$ and we will not enter into details here. However, it is important to stress that the magnetic field is taken as constant in the $z$ direction, what results in different contributions in the $r$ and $\theta$ directions when one calculates the magnetic field in spherical coordinates.

According to Ref., ${ }^{21,22}$ and assuming a perfectly conducting neutron star $\left(B_{r}(R)=0\right)$ that bears a magnetic dipole moment aligned with the rotation axis such that

$$
\mu=B_{p} R^{3} / 2
$$

where $R$ is the radius of the star and $B_{p}$ the magnetic field intensity at the pole, the components of the magnetic field in spherical coordinates are given by:

$$
B_{r}=B_{P} \cos \theta\left(\frac{R}{r}\right)^{3}, \quad B_{\theta}=\frac{B_{P}}{2} \sin \theta\left(\frac{R}{r}\right)^{3},
$$

and in this case, it is straightforward to show that $\nabla \cdot \mathbf{B}=0$.

If one cast eqs. (1) and (2) in spherical coordinates, from now on called respectively original and LL's prescriptions, they acquire the form $B_{r}=\cos \theta B_{z}$, $B_{\theta}=-\sin \theta B_{z}$ and $B_{\phi}=0$ and the resulting divergent reads

$$
\nabla \cdot \mathbf{B}=\cos \theta \frac{\partial B}{\partial r}
$$

where the magnetic field in the radial direction can be obtained from the solution of the TOV equations, ${ }^{23}$ where $r$ runs from the centre to the radius of the star. As a simple conclusion, $\nabla \cdot \mathbf{B}$ is generally not zero, except for some specific values of the parameters that we discuss in the next Section.

Let's emphasize that a recently published work $^{24}$ presents a beautiful selfconsistent solution of the space-time and electromagnetic field for the case of magnetised stars. This calculation also takes into account anisotropic effects by solving Einstein's field equation in a general relativistic formalism, but it still presents two flaws: 1) the values of the magnetic fields are quite reasonable in the central regions of the star, but at the surface their values are of the order of $10^{17} \mathrm{G}$, much too high as confronted with observational results and 2) it is computationally very demanding. We next we show a correct and simpler way to study magnetised neutron stars 
by simply avoiding the parameterisations of eqs. (1) and (2) that violate one of the Maxwell equations.

\section{Results and Discussion}

In what follows we analyse how much $\nabla \cdot \mathbf{B}$ deviates from zero when $B$ is allowed to vary either with the original prescription as in eq. (1) or with LL's proposal, as in eq. (2) with two different models, the NJL and the MIT bag model. These two models have been extensively used to describe stellar matter in the interior of quark stars. It is important to point out that the same test could be performed with hadronic models used to account for magnetised NS with hadronic constituents, as in Refs. ${ }^{7,8,10-}$, ${ }^{15,17,18}$ for instance.

Before we analyse the behaviour of $\nabla \cdot \mathbf{B} / B$ that depends on

$$
\frac{\partial B}{\partial r}=\frac{\partial B}{\partial n} \frac{\partial n}{\partial r}
$$

we show in Fig. 1 how the magnetic field varies with the star radius for one specific case, i.e., the MIT bag model with $B=10^{18} \mathrm{G}$ and the prescription given in eq.(2). All other cases studied next present a very similar behaviour. It is interesting to notice that the curve changes concavity around half the stellar radius.

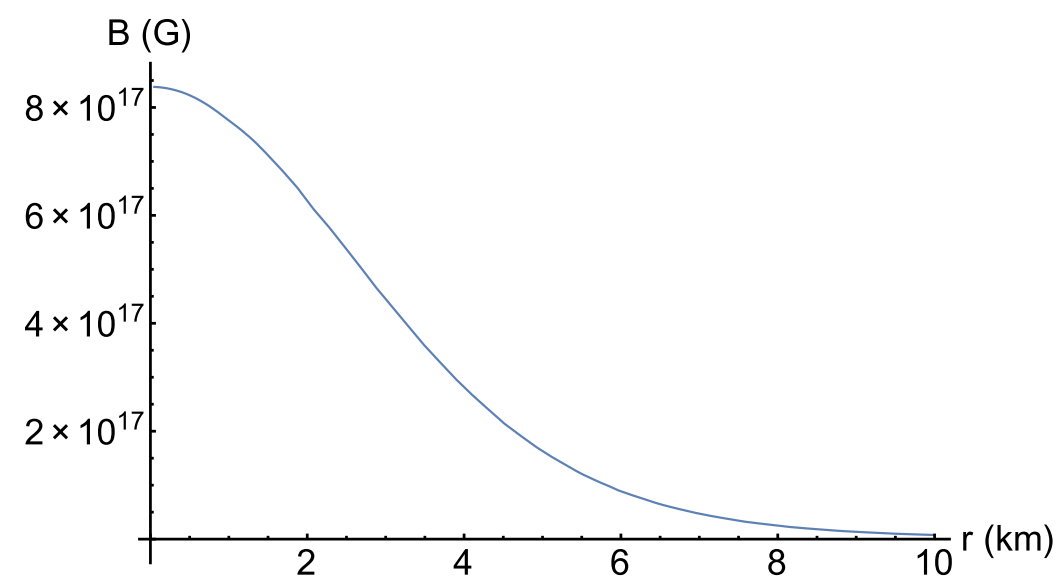

Fig. 1. Magnetic field versus $\mathrm{r}$ for the MIT model, bag constant $148 \mathrm{MeV}^{1 / 4}, M_{\max }=1.4 M_{\odot}$ and $R=10.04 \mathrm{~km}$.

In Fig. 2 we plot $\nabla \cdot \mathbf{B} / B$ as a function of the star radius for different latitude $(\theta)$ angles and a magnetic field equal to $B_{0}=10^{18} \mathrm{G}$. In both cases the equations of state were obtained with the Nambu-Jona-Lasinio model. ${ }^{9,25}$ The violation is quite strong and $\nabla \cdot \mathbf{B} / B$ can reach $70 \%$ for small angles.

In Figure 3 we plot the same quantity as in Figure 2 for the LL's prescription and the much simpler and also more used MIT bag model for different latitude 


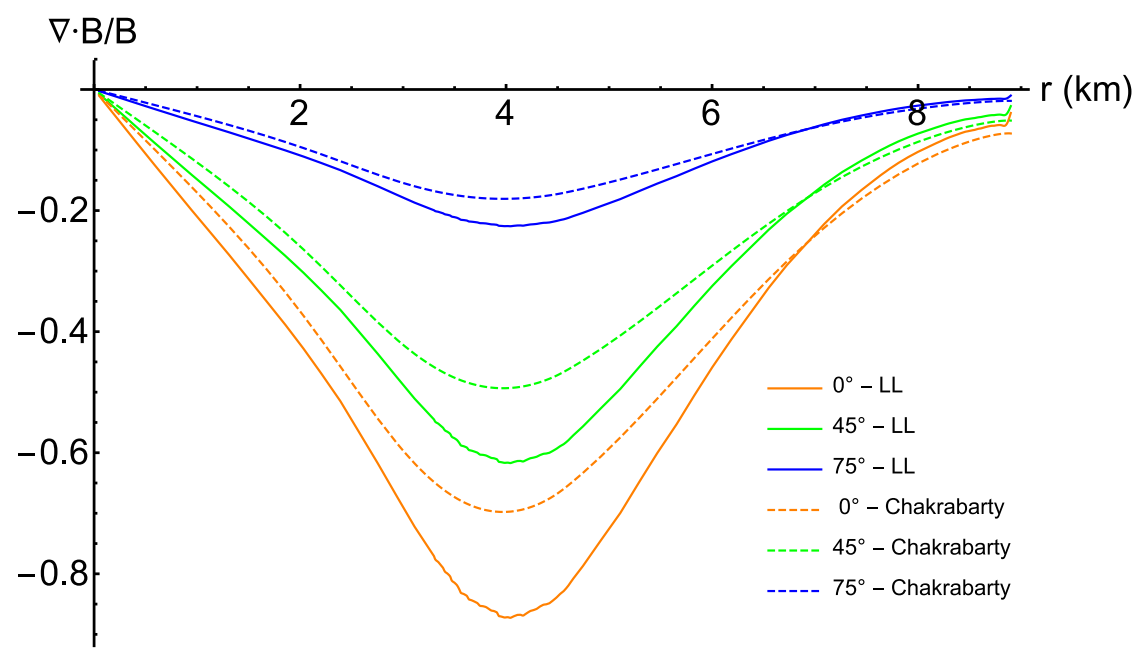

Fig. 2. Equations of state obtained with the NJL model and $B_{0}=10^{18}$ G. Chakrabarty's prescription was calculated with $M_{\max }=1.44 M_{\odot}$ and $R=8.88 \mathrm{~km}, \beta=5 \times 10^{-4}$ and $\gamma=3$. LL's prescription was calculated with $M_{\max }=1.46 M_{\odot}, R=8.83 \mathrm{~km}$ and $\zeta=3$.

angles. Again the violation amounts to the same values as the ones obtained within the NJL model. Finally, in Figure 4, we show how large the deviation can be for different values of the magnetic field intensity and a fixed angle $\theta=45$ degrees and the original prescription. We see that the deviation reaches approximately the same percentages, independently of the field intensity.

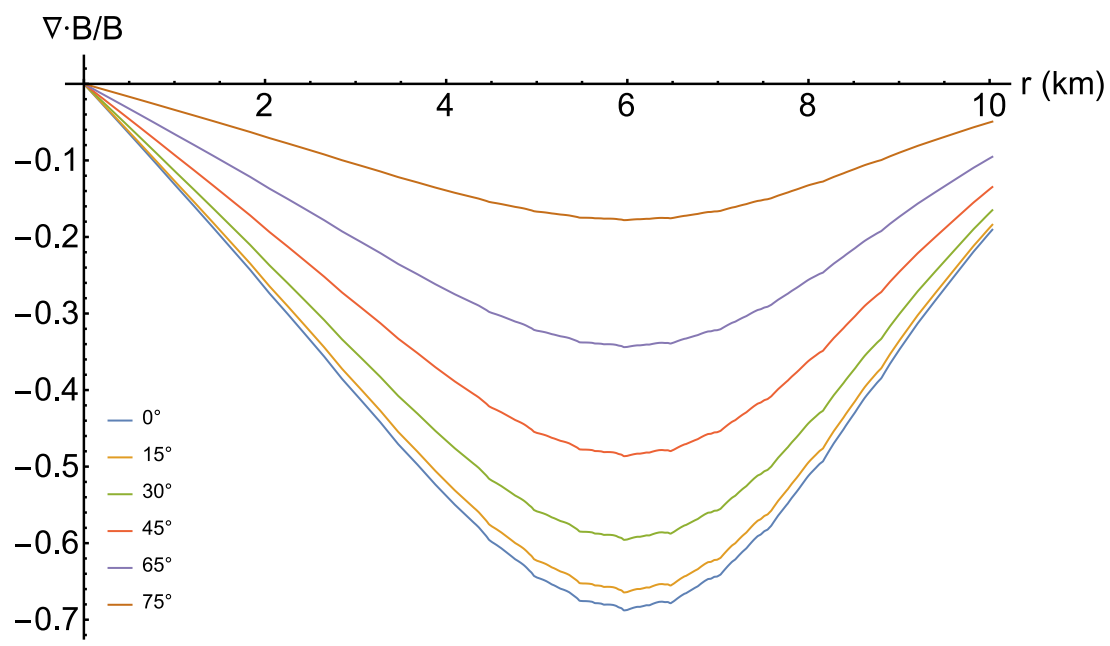

Fig. 3. Equations of state obtained with the MIT model, bag constant $148 \mathrm{MeV}^{1 / 4}, M_{\max }=$ $1.4 M_{\odot}$ and $R=10.04 \mathrm{~km}$ for different latitude angles. 


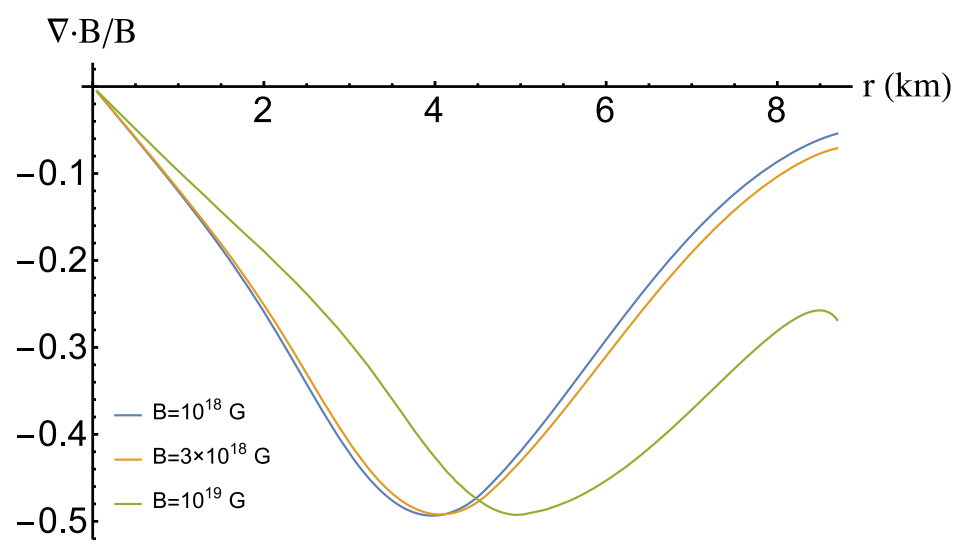

Fig. 4. Quark stars described by NJL model with different values of $B_{0}$ and $\theta=45$ degrees. For $B_{0}=10^{18} \mathrm{G}, M_{\max }=1.44 \odot, R=8.88 \mathrm{~km}$ and central energy density $\epsilon_{c}=7.67 \mathrm{fm}^{-4}$. For $B_{0}=3 \times 10^{18} \mathrm{G}: M_{\max }=1.45 \odot, R=8.88 \mathrm{~km}$ and central energy density $\epsilon_{c}=7.65 \mathrm{fm}^{-4}$. For $B_{0}=10^{19} G: M_{\max }=1.50_{\odot}, R=8.80 \mathrm{~km}$ and central energy density $\epsilon_{c}=8.11 \mathrm{fm}^{-4}$.

Now, we turn our attention to a possible generalisation of eqs. (1) and (2) in spherical coordinates in order to verify for which conditions the divergent becomes zero and check whether the situation can be circumvented. The generalized magnetic field components is given by

$$
B_{r}=B_{0} \cos \theta(f(r))^{\eta}, \quad B_{\theta}=-\frac{B_{0}}{\zeta} \sin \theta(f(r))^{\eta},
$$

and the corresponding divergent reads:

$$
\nabla \cdot \mathbf{B}=B_{0} \cos \theta\left[\frac{2 f(r)^{\eta}}{r}+\eta f(r)^{\eta-1} \frac{d f}{d r}-\frac{2 f(r)^{\eta}}{r \zeta}\right],
$$

which is zero either for the trivial solution $\cos \theta=0$ or if

$$
\frac{2}{r}+\frac{\eta}{f(r)} \frac{d f}{d r}-\frac{2}{r \zeta}=0
$$

for which a general solution has the form

$$
f(r)=A r^{\frac{2-2 \zeta}{\eta \zeta}} .
$$

When $\zeta=-2$ and $\eta=3, A=R$ and $B_{0}=B_{p}$, eqs.(3) are recovered. When $\zeta=1$, the numerator of the exponent becomes zero and $f(r)$ is simply a constant $(A)$, does not depending on $r$. Another possibility is the assumption that $f(r)$ is a function of the density, for instance, as $f(n(r))$ or of the energy density as $f(\varepsilon(r))$. In these cases,

$$
\frac{d f}{d n} \frac{d n}{d r}=\frac{2 f}{r \eta}\left(\frac{1-\zeta}{\zeta}\right), \quad \frac{d f}{d \varepsilon} \frac{d \varepsilon}{d r}=\frac{2 f}{r \eta}\left(\frac{1-\zeta}{\zeta}\right) .
$$


If we take $\zeta=1$, the result resembles the original (LL's) prescription, $\frac{d f}{d n}=0$ $\left(\frac{d f}{d \varepsilon}=0\right)$ because $\frac{d n}{d r}\left(\frac{d \varepsilon}{d r}\right)$ is obtained from the TOV equations and is never zero. For $\zeta \neq 1$, solutions can be obtained from numerical integration.

\section{Final Remarks}

We have shown that both existent prescriptions for a density dependent magnetic field widely used in the study of magnetised neutron star matter equations of state strongly violate one of the Maxwell equations, showing a pathological problem. However, they can be cured if used only for well defined values of functionals that guarantee that the Maxwell equation is not violated. We have shown the results obtained from EOS used to describe quarks stars. Had we shown the same quantities for neutron stars constituted of hadronic matter, the qualitative results would be the same. As a final word of caution, we would like to comment that the divergence problem in general relativity goes beyond the simple analysis we have performed and will deserve an attentive look in the future.

\section{Acknowledgments}

This work was partially supported by CNPq under grant 300602/2009-0. We would like to thank very fruitful discussions with Prof. Constança Providência.

\section{References}

1. R. C. Duncan and C. Thompson, Astrophys. J. 392, L9 (1992); R. C. Duncan and C. Thompson, Mon. Not. R. Astron. Soc. 275, 255 (1995); R. C. Duncan, C. Thompson, Astrophys. J. 473, 322 (1996).

2. V. V. Usov, Millisecond Pulsars with Extremely Strong Magnetic Fields as a Cosmological Source of $\gamma$-ray Bursts, Nature 357, 472 (1992).

3. L. G. Spitler et al, Nature 531, 202 (2016).

4. D. Bandyopadhyay, S. Chakrabarty, and S. Pal, Phys. Rev. Lett. 79, 2176 (1997).

5. S. Chakrabarty, Phys. Rev. D 54, 1306 (1996).

6. A. Broderick, M. Prakash, and J. M. Lattimer, Astrophys. J. 537, 351 (2000).

7. G. J. Mao, C. J. Mao, A. Iwamoto, and Z. X. Li, Chin. J. Astron. Astrophys. 3, 359 (2003).

8. A. Rabhi et al., J. Phys. G 36, 115204 (2009).

9. D. P. Menezes et al, Phys. Rev. C 79, 035807 (2009); D. P. Menezes et al., Phys. Rev. C 80, 065805 (2009).

10. C. Y. Ryu, K. S. Kim, and M. Ki Cheoun, Phys. Rev. C 82, 025804 (2010).

11. A. Rabhi, P. K. Panda, and C. Providencia, Phys. Rev. C84, 035803 (2011).

12. R. Mallick and M. Sinha, Mon. Not. R. Astron. Soc. 414, 2702 (2011).

13. L. L. Lopes and D. P. Menezes, Braz. J. Phys. 42, 428 (2012).

14. V. Dexheimer, R. Negreiros, and S. Schramm, Eur. J. Phys. A 48, 189 (2012).

15. R. H. Casali, L. B. Castro, and D. P. Menezes, Phys. Rev. C 89, 015805 (2014).

16. D. P. Menezes et al., Phys. Rev. C 89, 055207 (2014).

17. R. Mallick and S. Schramm, Phys. Rev. C 89, 045805 (2014).

18. R. O. Gomes, V. Dexheimer, and C. A. Z. Vasconcellos, Astron. Nachr. 335, 666 (2014). 
19. V. Dexheimer, D. P. Menezes, and M. Strickland, J. Phys. G 41, 015203 (2014).

20. L. L. Lopes and D. Menezes, J. Cosmology and Astroparticle Physics 08, 002 (2015).

21. D. Viganò, Magnetic Fields in Neutron Stars, Ph.D. Thesis, Universidad de Alicante, Spain, 2013.

22. P. Goldreich and W. H. Julian, ApJ 157, 869 (1969).

23. R. C. Tolman, Phys. Rev. 55, 364 (1939); J. R. Oppenheimer and G. M. Volkoff, Phys. Rev. 55, 374 (1939).

24. D. Chatterjee et al., MNRAS, V. 447, Issue 4, 3785 (2015).

25. D. P. Menezes and L. L. Lopes, Eur. Phys. J. A 52, 17 (2016). 